\title{
WEAKLY TIGHT FUNCTIONS AND THEIR DECOMPOSITION
}

\author{
MONA KHARE AND BHAWNA SINGH
}

Received 2 September 2004 and in revised form 30 December 2004

The present paper deals with the study of a weakly tight function and its relation to tight functions. We obtain a Jordan-decomposition-type theorem for a locally bounded weakly tight real-valued function defined on a sublattice of $I^{X}$, followed by the notion of a total variation.

\section{Introduction}

The notion of a signed measure arises if a measure is allowed to take on both positive and negative values. A set that is both positive and negative with respect to a signed measure is termed as a null set. Some concepts in measure theory can be generalized by means of classes of null sets. An abstract formulation and proof of the Lebesgue decomposition theorem using the concept of null sets is given by Ficker [5]. A real-valued function satisfying certain properties that can be expressed as a difference of two nonnegative functions possessing the same properties is called "decomposable." Several Jordan-decompositiontype theorems are exhibited in [3]. Faires and Morrison [4] exposed conditions on a vector-valued measure that ensure vector-valued Jordan-decomposition-type theorem to hold. For a signed null-additive fuzzy measure, a Jordan-decomposition-type theorem is investigated by Pap in [11].

The problem of generation of measures by tight functions defined on a lattice of sets has been taken up by several authors $[1,2,6,8,9]$. Nayak and Srinivasan [10] initiated a weaker form of tightness for a real-valued function $\mu$ defined on a lattice of sets to decompose $\mu$ as a difference $\mu^{+}-\mu^{-}$and then extended it to a countably additive measure.

In Section 2, we have defined and studied the notions of measuring envelopes, modular functions, and additive functions. The notions of superadditive and subadditive functions are also given with the help of pointwise addition of elements in $I^{X}$. The lower envelope $\beta_{*}$ of a superadditive function $\beta$ defined on a sublattice $K$ of $I^{X}$ turns out to be superadditive. In Section 3, we introduce the notion of a weakly tight function $\beta: K \rightarrow \mathbb{R}$, where $K$ is a sublattice of $I^{X}$ containing 0 and 1 (cf. [10]). The condition imposed on the $[0,1]$-valued function $\beta$ to be a weakly tight function is less restrictive than that for being a tight function. It is proved that a superadditive, monotone, and weakly tight function 
$\beta$ is tight (there $K$ is taken to be closed under addition). In Section 4, our main result states that a locally bounded, weakly tight real-valued function $\beta$ defined on $K$ has a representation of the form $\beta^{+}-\beta^{-}$; both $\beta^{+}$and $\beta^{-}$are nonnegative monotone (and hence, locally bounded) functions defined on $K$. If, in addition, $\beta$ is additive and modular, then the decomposed parts $\beta^{+}$and $\beta^{-}$preserve superadditive and supermodular properties. The total variation $|\beta|$ of $\beta$ is defined as the sum of $\beta^{+}$and $\beta^{-}$, following the terminology in the classical measure theory (cf. [12]); few properties of $|\beta|$ are noted.

Notations. Throughout this paper, $X$ is a nonempty set and $I \equiv[0,1]$ is the unit interval of the real line $\mathbb{R} ; C$ denotes a subfamily of $I^{X}$ of all functions from $X$ to $I ; K$ stands for a sublattice of $I^{X}$ containing the least element 0 and the greatest element 1 , where 0 and 1 are constant functions sending each $x \in X$ to 0 and 1 , respectively. We will denote by $\beta$ a function from $K$ to $I$ satisfying $\beta(0)=0$.

\section{Measuring envelopes}

Let $C$ be a sublattice of $I^{X}$ and let $\xi: C \rightarrow I$ be a function. Then $\xi$ is called monotone if $f$, $g \in C, g \leq f \Rightarrow \xi(g) \leq \xi(f)$. The mapping $\xi$ is called supermodular (submodular, resp.) if for $f, g \in C, \xi(f)+\xi(g) \leq \xi(f \vee g)+\xi(f \wedge g)(\xi(f)+\xi(g) \geq \xi(f \vee g)+\xi(f \wedge g)$, resp. $)$; $\xi$ is said to be modular if it is both supermodular and submodular. The mapping $\xi$ is said to be superadditive (subadditive, resp.) if for $f_{1}, f_{2} \in C$ such that $f_{1}+f_{2} \in C, \xi\left(f_{1}+\right.$ $\left.f_{2}\right) \geq \xi\left(f_{1}\right)+\xi\left(f_{2}\right)\left(\xi\left(f_{1}+f_{2}\right) \leq \xi\left(f_{1}\right)+\xi\left(f_{2}\right)\right.$, resp. $) ; \xi$ is said to be additive if it is both superadditive and subadditive. If we restrict ourselves to disjoint crisp sets in $C$, then the condition of being additive for $\xi$ coincides with that of [10].

The family $C \subseteq I^{X}$ is said to be closed under addition if for $f, g \in C$ with $f+g \in I^{X}$, we have $f+g \in C$, and is said to be closed under addition modulo 1 (or closed under $\oplus$ ) if $f, g \in C$ implies that $f \oplus g \in C$, where $f \oplus g=(f+g) \wedge 1$.

A function $\xi: C \rightarrow I$ is called subadditive modulo 1 if for $f_{1}, f_{2} \in C$ with $f_{1} \oplus f_{2} \in C$, we have $\xi\left(f_{1} \oplus f_{2}\right) \leq \xi\left(f_{1}\right)+\xi\left(f_{2}\right)$; here $C \subseteq I^{X}$.

If $f, g \in I^{X}$ and $f+g \in I^{X}$, then $f \oplus g=f+g$. Thus if $C$ is closed under addition modulo 1 , then $C$ is closed under addition. Also, if $\xi$ is subadditive modulo 1 , then $\xi$ is subadditive.

The definition for a function $\xi: C \rightarrow I$ to be supermodular (submodular, superadditive, subadditive, resp.) continues to hold for a real-valued function $\xi$ defined on $C$.

Definition 2.1. Let $\beta: K \rightarrow I$ be a function satisfying $\beta(0)=0$. Define $\beta_{*}: I^{X} \rightarrow I$ and $\beta^{*}: I^{X} \rightarrow I$ by

$$
\begin{aligned}
& \beta_{*}(f)=\sup \{\beta(g): g \leq f, g \in K\}, \\
& \beta^{*}(f)=\inf \{\beta(g): g \geq f, g \in K\}, \quad f \in I^{X} .
\end{aligned}
$$

$\beta_{*}$ and $\beta^{*}$ are called the lower envelope and the upper envelope of $\beta$, respectively.

We obtain

(i) $\beta_{*}(0)=0=\beta^{*}(0)$;

(ii) both $\beta_{*}$ and $\beta^{*}$ are monotone; 
(iii) $\beta^{*}\left|K \leq \beta \leq \beta_{*}\right| K$;

(iv) $\beta$ is monotone if and only if $\beta_{*}\left|K=\beta=\beta^{*}\right| K$.

Proposition 2.2. (i) If $\beta$ is supermodular, then $\beta_{*}$ is supermodular.

(ii) If $\beta$ is submodular, then $\beta^{*}$ is submodular.

Proof. (i) Let $f_{1}, f_{2} \in I^{X}$. Let $\varepsilon>0$. Then there exist $g_{1}, g_{2} \in K, f_{1} \geq g_{1}, f_{2} \geq g_{2}$ such that

$$
\begin{aligned}
& \beta_{*}\left(f_{1}\right)-\frac{\varepsilon}{2}<\beta\left(g_{1}\right), \\
& \beta_{*}\left(f_{2}\right)-\frac{\varepsilon}{2}<\beta\left(g_{2}\right) .
\end{aligned}
$$

It follows that

$$
\beta_{*}\left(f_{1}\right)+\beta_{*}\left(f_{2}\right)-\varepsilon<\beta\left(g_{1} \vee g_{2}\right)+\beta\left(g_{1} \wedge g_{2}\right)
$$

Consequently,

$$
\beta_{*}\left(f_{1}\right)+\beta_{*}\left(f_{2}\right)-\varepsilon<\beta_{*}\left(f_{1} \vee f_{2}\right)+\beta_{*}\left(f_{1} \wedge f_{2}\right) .
$$

Since $\varepsilon$ is arbitrary, we get

$$
\beta_{*}\left(f_{1}\right)+\beta_{*}\left(f_{2}\right) \leq \beta_{*}\left(f_{1} \vee f_{2}\right)+\beta_{*}\left(f_{1} \wedge f_{2}\right) .
$$

Proof of (ii) follows analogously.

Proposition 2.3. (i) If $K$ is closed under addition and $\beta$ is superadditive, then $\beta_{*}$ is superadditive.

(ii) If $K$ is closed under addition modulo 1 and $\beta$ is subadditive modulo 1 , then $\beta^{*}$ is subadditive modulo 1 , and hence $\beta^{*}$ is subadditive.

Proof. (i) Let $f_{1}$ and $f_{2}$ be in $I^{X}$ such that $f_{1}+f_{2} \in I^{X}$. Let $\varepsilon>0$. Then there exist $g_{1}, g_{2} \in K$ with $g_{1} \leq f_{1}$ and $g_{2} \leq f_{2}$ such that $\beta\left(g_{1}\right)>\beta_{*}\left(f_{1}\right)-\varepsilon / 2$ and $\beta\left(g_{2}\right)>\beta_{*}\left(f_{2}\right)-\varepsilon / 2$. Hence, $\beta_{*}\left(f_{1}\right)+\beta_{*}\left(f_{2}\right)-\varepsilon<\beta\left(g_{1}\right)+\beta\left(g_{2}\right)$. Since, for any $x \in X, 0 \leq\left(g_{1}+g_{2}\right)(x) \leq\left(f_{1}+f_{2}\right)(x) \leq$ 1 , we get $g_{1}+g_{2} \in I^{X}$ and so $g_{1}+g_{2} \in K$. Since $\beta$ is superadditive, $\beta_{*}\left(f_{1}\right)+\beta_{*}\left(f_{2}\right)-\varepsilon<$ $\beta\left(g_{1}+g_{2}\right) \leq \beta_{*}\left(f_{1}+f_{2}\right)$ and hence, we obtain $\beta_{*}\left(f_{1}\right)+\beta_{*}\left(f_{2}\right) \leq \beta_{*}\left(f_{1}+f_{2}\right)$.

(ii) Let $f_{1}, f_{2} \in I^{X}$. Let $\varepsilon>0$. Then there exist $g_{1}, g_{2} \in K$ with $f_{1} \leq g_{1}$ and $f_{2} \leq g_{2}$ such that

$$
\beta^{*}\left(f_{1}\right)+\beta^{*}\left(f_{2}\right)+\varepsilon>\beta\left(g_{1}\right)+\beta\left(g_{2}\right) .
$$

Since $g_{1}, g_{2} \in K$ and $K$ is closed under $\oplus$, we get $g_{1} \oplus g_{2} \in K$. Also, $f_{1} \oplus f_{2} \leq g_{1} \oplus g_{2}$. Hence (2.6) gives

$$
\beta^{*}\left(f_{1}\right)+\beta^{*}\left(f_{2}\right)+\varepsilon>\beta\left(g_{1} \oplus g_{2}\right) \geq \beta^{*}\left(f_{1} \oplus f_{2}\right) .
$$

Since $\varepsilon$ is arbitrary, we obtain that $\beta^{*}$ is subadditive modulo 1 . 
Definitions 2.4. Let $\beta_{1}$ and $\beta_{2}$ be real-valued functions defined on $K$. Define $\left(\beta_{1}+\beta_{2}\right)(f)=$ $\beta_{1}(f)+\beta_{2}(f), f \in K$, and $\left(\beta_{1}-\beta_{2}\right)(f)=\beta_{1}(f)-\beta_{2}(f), f \in K$. Likewise, for $\beta: K \rightarrow \mathbb{R}$ and $\lambda \in \mathbb{R}$, define $(\lambda \beta)(f)=\lambda \beta(f), f \in K$.

Proposition 2.5. (i) If $\beta_{1}$ and $\beta_{2}$ are supermodular (submodular, resp.), then $\beta_{1}+\beta_{2}$ is supermodular (submodular, resp.).

If both $\beta_{1}$ and $\beta_{2}$ are modular, then so are $\beta_{1}+\beta_{2}$ and $\beta_{1}-\beta_{2}$.

(ii) If $\beta$ is supermodular (submodular, resp.) then $\lambda \beta$ is supermodular (submodular, resp.), where $\lambda$ is a nonnegative real number.

(iii) If $K$ is closed under addition, and $\beta_{1}, \beta_{2}$ are superadditive (subadditive, resp.), then $\beta_{1}+\beta_{2}$ is superadditive (subadditive, resp.). If both $\beta_{1}$ and $\beta_{2}$ are additive, then so are $\beta_{1}+\beta_{2}$ and $\beta_{1}-\beta_{2}$.

(iv) If $K$ is closed under addition, and $\beta$ is superadditive (subadditive, resp.), then $\lambda \beta$ is superadditive (subadditive, resp.), where $\lambda$ is a nonnegative real number.

Proof. We will prove only (i) and (iii).

(i) Let $\beta_{1}$ and $\beta_{2}$ be supermodular. Let $f, g \in K$. Then,

$$
\begin{aligned}
\left(\beta_{1}+\beta_{2}\right)(f)+\left(\beta_{1}+\beta_{2}\right)(g) & =\beta_{1}(f)+\beta_{2}(f)+\beta_{1}(g)+\beta_{2}(g) \\
& \leq \beta_{1}(f \vee g)+\beta_{1}(f \wedge g)+\beta_{2}(f \vee g)+\beta_{2}(f \wedge g) \\
& =\left(\beta_{1}+\beta_{2}\right)(f \vee g)+\left(\beta_{1}+\beta_{2}\right)(f \wedge g) .
\end{aligned}
$$

If $\beta_{1}$ and $\beta_{2}$ are submodular, then, by similar arguments, $\beta_{1}+\beta_{2}$ is submodular.

(iii) Let $K$ be closed under addition. Let $\beta_{1}$ and $\beta_{2}$ be superadditive. Let $f, g \in K$. Then,

$$
\begin{aligned}
\left(\beta_{1}+\beta_{2}\right)(f)+\left(\beta_{1}+\beta_{2}\right)(g) & =\beta_{1}(f)+\beta_{2}(f)+\beta_{1}(g)+\beta_{2}(g) \\
& \leq \beta_{1}(f+g)+\beta_{2}(f+g)=\left(\beta_{1}+\beta_{2}\right)(f+g) .
\end{aligned}
$$

\section{Weakly tight functions}

Definition 3.1. Let $\beta: K \rightarrow I$ with $\beta(0)=0$. Then $\beta$ is called tight (cotight, resp.) if

$$
\begin{gathered}
\beta\left(f_{2}\right)=\beta\left(f_{1}\right)+\beta_{*}\left(f_{2}-f_{1}\right), \quad f_{1}, f_{2} \in K, f_{1} \leq f_{2}, \\
\left(\beta\left(f_{2}\right)=\beta\left(f_{1}\right)+\beta^{*}\left(f_{2}-f_{1}\right), f_{1}, f_{2} \in K, f_{1} \leq f_{2}, \text { resp. }\right) .
\end{gathered}
$$

If $\beta$ is tight (or cotight), then $\beta$ is modular and monotone. Furthermore, if $\beta$ is tight (cotight, resp.), then $\beta_{*}\left(\beta^{*}\right.$, resp.) is an extension of $\beta$. A detailed study of tight and cotight functions is made in $[7,13]$.

Definition 3.2. Let $\beta: K \rightarrow \mathbb{R}$ be a function with $\beta(0)=0$. Then $\beta$ is called weakly tight if for every pair $f_{1}, f_{2} \in K$ with $f_{1} \leq f_{2}$ and for any $\varepsilon>0$, there exists $f \in K$ such that $f \leq f_{2}-f_{1}$ and

$$
\left|\beta\left(f_{2}\right)-\beta\left(f_{1}\right)-\beta(f)\right|<\varepsilon
$$


Proposition 3.3. Let $\beta: K \rightarrow I$ be a function satisfying $\beta(0)=0$. If $\beta$ is tight, then $\beta$ is weakly tight.

Proof. Let $f_{1}, f_{2} \in K$ with $f_{1} \leq f_{2}$. Let $\varepsilon>0$. Since $\beta$ is tight, there exists $f \in K$ with $f \leq$ $f_{2}-f_{1}$ such that

$$
\beta_{*}\left(f_{2}-f_{1}\right)-\varepsilon=\beta\left(f_{2}\right)-\beta\left(f_{1}\right)-\varepsilon<\beta(f)
$$

or

$$
\beta\left(f_{2}\right)-\beta\left(f_{1}\right)-\beta(f)<\varepsilon
$$

Since $\beta$ is monotone and $f \leq f_{2}-f_{1}$, we obtain

$$
\beta(f)=\beta_{*}(f) \leq \beta_{*}\left(f_{2}-f_{1}\right)=\beta\left(f_{2}\right)-\beta\left(f_{1}\right),
$$

and so

$$
\beta\left(f_{2}\right)-\beta\left(f_{1}\right)-\beta(f) \geq 0 \text {. }
$$

Thus, $\left|\beta\left(f_{2}\right)-\beta\left(f_{1}\right)-\beta(f)\right|<\varepsilon$.

Proposition 3.4. Let $K$ be closed under addition. Let $\beta: K \rightarrow I$ be superadditive, monotone, and weakly tight. Then $\beta$ is tight.

Proof. Let $f_{1}, f_{2} \in K$ with $f_{1} \leq f_{2}$. Let $\varepsilon>0$. Since $\beta$ is weakly tight, there exists $f \in K$ such that $f \leq f_{2}-f_{1}$ and

$$
\left|\beta\left(f_{2}\right)-\beta\left(f_{1}\right)-\beta(f)\right|<\varepsilon
$$

Consequently,

$$
\beta\left(f_{2}\right)-\beta\left(f_{1}\right)<\beta(f)+\varepsilon \leq \beta_{*}\left(f_{2}-f_{1}\right)+\varepsilon .
$$

Since $\varepsilon$ is arbitrary, we get $\beta\left(f_{2}\right)-\beta\left(f_{1}\right) \leq \beta_{*}\left(f_{2}-f_{1}\right)$.

Since, by Proposition 2.3(i), $\beta_{*}$ is superadditive, we get $\beta\left(f_{2}\right)=\beta_{*}\left(f_{2}\right) \geq \beta_{*}\left(f_{2}-f_{1}\right)+$ $\beta_{*}\left(f_{1}\right)$, which yields that $\beta_{*}\left(f_{2}-f_{1}\right) \leq \beta_{*}\left(f_{2}\right)-\beta_{*}\left(f_{1}\right)=\beta\left(f_{2}\right)-\beta\left(f_{1}\right)$.

Thus $\beta_{*}\left(f_{2}-f_{1}\right)=\beta\left(f_{2}\right)-\beta\left(f_{1}\right)$, that is, $\beta$ is tight.

\section{A Jordan-decomposition-type theorem}

In this section, $\beta$ is a real-valued function defined on a sublattice $K$ of $I^{X}$ containing 0 and 1. Also, it is assumed throughout this section that $\beta$ is locally bounded, that is, for any $f$ in $K, \sup \{\beta(g): g \leq f, g \in K\}$ exists. For a locally bounded real-valued function $\beta$, the definitions of lower and upper envelopes, $\beta_{*}$ and $\beta^{*}$, of $\beta$ may be given in the same way.

Definition 4.1. For $f \in K$, define $\beta^{+}(f)$ and $\beta^{-}(f)$ as follows:

$$
\begin{aligned}
& \beta^{+}(f)=\sup \{\beta(g): g \leq f, g \in K\}, \\
& \beta^{-}(f)=-\inf \{\beta(g): g \leq f, g \in K\} .
\end{aligned}
$$


2996 Weakly tight functions and their decomposition

Remarks 4.2. (i) $\beta^{+}=\beta_{*} \mid K$.

(ii) $\beta^{-}=(-\beta)^{+} ; \beta^{+}=(-\beta)^{-}$.

(iii) Both $\beta^{+}$and $\beta^{-}$are nonnegative, monotone (and hence, locally bounded).

(iv) $-\beta^{-} \leq \beta \leq \beta^{+}$.

THEOREM 4.3. Let $\beta$ be a weakly tight real-valued function defined on $K$. Then $\beta=\beta^{+}-\beta^{-}$. Proof. Let $\varepsilon>0$. Let $f \in K$. Then there exists $f_{1} \in K$ such that $f_{1} \leq f$ and

$$
\beta^{+}(f)<\beta\left(f_{1}\right)+\frac{\varepsilon}{2}
$$

Since $\beta$ is weakly tight, there exists $f_{2} \in K$ such that $f_{2} \leq f-f_{1}$ and

$$
\left|\beta(f)-\left(\beta\left(f_{1}\right)+\beta\left(f_{2}\right)\right)\right|<\frac{\varepsilon}{2} .
$$

This implies that

$$
\beta\left(f_{1}\right)+\beta\left(f_{2}\right)<\beta(f)+\frac{\varepsilon}{2} .
$$

Also, $f_{2} \leq f-f_{1} \leq f$ yields that $-\beta^{-}(f) \leq \beta\left(f_{2}\right)$. Hence, using (4.2) and (4.4), we get $\beta^{+}(f)-\beta^{-}(f)<\beta\left(f_{1}\right)+\varepsilon / 2+\beta\left(f_{2}\right)<\beta(f)+\varepsilon$. Since $\varepsilon$ is arbitrary, we get

$$
\beta^{+}-\beta^{-} \leq \beta
$$

Replacing $\beta$ in (4.5) by $-\beta$, we get

$$
(-\beta)^{+}-(-\beta)^{-} \leq-\beta
$$

or

$$
\beta^{-}-\beta^{+} \leq-\beta
$$

or

$$
\beta^{+}-\beta^{-} \geq \beta
$$

Thus, $\beta^{+}-\beta^{-}=\beta$.

Proposition 4.4. Let $K$ be closed under addition. If $\beta$ is additive, then both $\beta^{+}$and $\beta^{-}$are superadditive.

Proof. The proof follows from Proposition 2.3(i).

Proposition 4.5. If $\beta$ is modular, then both $\beta^{+}$and $\beta^{-}$are supermodular.

Proof. The proof follows from Proposition 2.2(i).

The results obtained in this section may be summarized as follows. 
Theorem 4.6 (Jordan-decomposition-type theorem). Let $K$ be a sublattice of $I^{X}$ containing 0 and 1 . If $\beta: K \rightarrow \mathbb{R}$ is locally bounded and weakly tight, then $\beta$ can be written as

$$
\beta=\beta^{+}-\beta^{-}
$$

where both $\beta^{+}$and $\beta^{-}$are nonnegative and monotone (and hence, locally bounded) functions defined on $K$. Furthermore, if $\beta$ is modular ( $\beta$ is additive and $K$ is closed under addition), then the decomposed parts $\beta^{+}$and $\beta^{-}$are supermodular (superadditive).

Definition 4.7. For a function $\beta: K \rightarrow \mathbb{R}$, define the total variation of $\beta$, written as $|\beta|$, by

$$
|\beta|=\beta^{+}+\beta^{-} .
$$

Theorem 4.8. Let $\beta: K \rightarrow \mathbb{R}$ be a locally bounded function.

(i) If $\beta$ is weakly tight, then $\beta=0 \Leftrightarrow|\beta|=0$.

(ii) For each $f \in K,|\beta(f)| \leq|\beta|(f)$.

(iii) If $\beta$ is modular, then $|\beta|$ is supermodular.

(iv) Let $K$ be closed under addition. Then $\beta$ being additive implies that $|\beta|$ is superadditive.

Proof. (i) If $\beta=0$, then $\beta^{+}=0=\beta^{-}$and so $|\beta|=0$. Conversely, if $|\beta|=0$, then both $\beta^{+}$ and $\beta^{-}$vanish. Since $\beta$ is weakly tight, by Theorem $4.3, \beta=\beta^{+}-\beta^{-}$. Hence $\beta=0$.

(ii) Let $f \in K$. Then by Remark 4.2(iv), $\beta(f) \leq \beta^{+}(f)$, and $-\beta(f) \leq \beta^{-}(f)$.

If $\beta(f)>0$, then

$$
|\beta|(f)=\beta^{+}(f)+\beta^{-}(f) \geq \beta(f)=|\beta(f)| .
$$

If $\beta(f)<0$, then

$$
|\beta(f)|=-\beta(f) \leq \beta^{-}(f) \leq|\beta|(f)
$$

(iii) Follows from Proposition 4.5 and Proposition 2.5(i).

(iv) Follows from Proposition 4.4 and Proposition 2.5(iii).

Remark 4.9. If we restrict ourselves to $\{0,1\}$-valued functions in $K$, then $K$ may be viewed as a sublattice of $P(X)$. For a $[0, \infty]$-valued function $\beta$ defined on this restricted $K$ over $P(X)$, the definitions of lower and upper envelopes, $\beta_{*}$ and $\beta^{*}$, reduce to the corresponding definitions in classical theory given by Adamski [1]. In this manner, the present study generalizes the theory in [1].

\section{References}

[1] W. Adamski, Tight set functions and essential measure, Measure Theory, Oberwolfach 1981 (Oberwolfach, 1981) (D. Kölzow and D. Maharam-Stone, eds.), Lecture Notes in Math., vol. 945, Springer, Berlin, 1982, pp. 1-14.

[2] J. R. Choksi, On compact contents, J. London Math. Soc. 33 (1958), 387-398.

[3] J. Diestel and B. Faires, On vector measures, Trans. Amer. Math. Soc. 198 (1974), 253-271.

[4] B. Faires and T. J. Morrison, The Jordan decomposition of vector-valued measures, Proc. Amer. Math. Soc. 60 (1976), 139-143. 


\section{Weakly tight functions and their decomposition}

[5] V. Ficker, An abstract formation of the Lebesgue decomposition theorem, Proc. Amer. Math. Soc. 60 (1976), 101-105.

[6] J. L. Kelley and T. P. Srinivasan, Pre-measures on lattices of sets, Math. Ann. 190 (1970/1971), 233-241.

[7] M. Khare and B. Singh, Cotight functions and extension to quasi*-measure, in preparation.

[8] J. Kisyński, On the generation of tight measures, Studia Math. 30 (1968), 141-151.

[9] P. Morales, Extension of a tight set function with values in a uniform semigroup, Measure Theory, Oberwolfach 1981 (Oberwolfach, 1981) (D. Kölzow and D. Maharam-Stone, eds.), Lecture Notes in Math., vol. 945, Springer, Berlin, 1982, pp. 282-290.

[10] M. K. Nayak and T. P. Srinivasan, Scalar and vector valued premeasures, Proc. Amer. Math. Soc. 48 (1975), 391-396.

[11] E. Pap, Null-Additive Set Functions, Mathematics and Its Applications, vol. 337, Kluwer Academic Publishers, Dordrecht, 1995.

[12] H. L. Royden, Real Analysis, 3rd ed., Macmillan Publishing, New York, 1981.

[13] P. Srivastava, M. Khare, and B. Singh, Premeasure spaces, tight functions and extension to quasi*measure, in preparation.

Mona Khare: Department of Mathematics and Statistics, Faculty of Science, University of Allahabad, Allahabad 211002, Uttar Pradesh, India

E-mail address: raghav9802@rediffmail.com

Bhawna Singh: Allahabad Mathematical Society, 10 CSP Singh Marg, Allahabad 211001, Uttar Pradesh, India

E-mail address: bsingh2@gmu.edu 


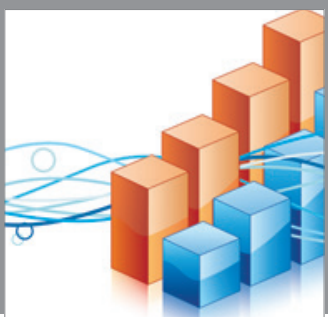

Advances in

Operations Research

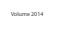

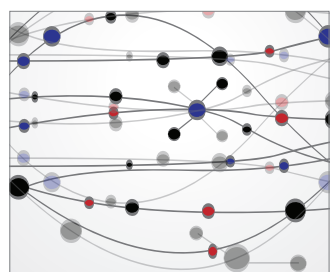

\section{The Scientific} World Journal
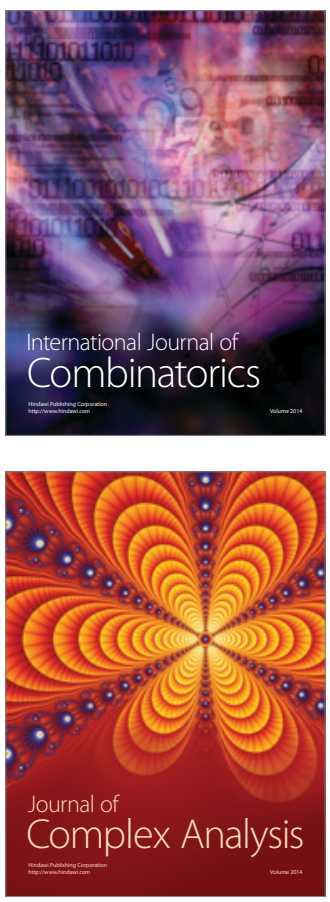

International Journal of

Mathematics and

Mathematical

Sciences
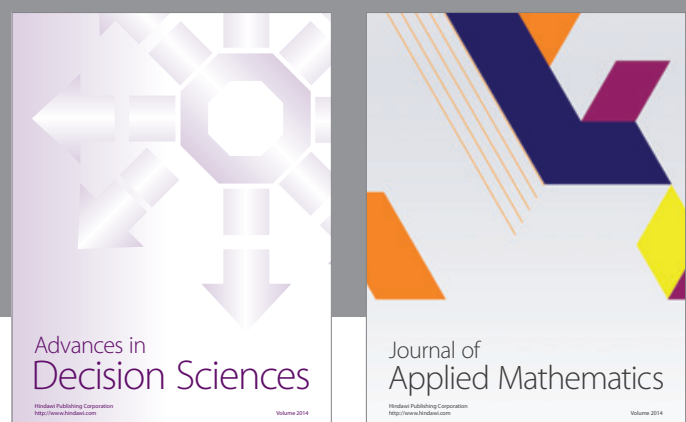

Journal of

Applied Mathematics
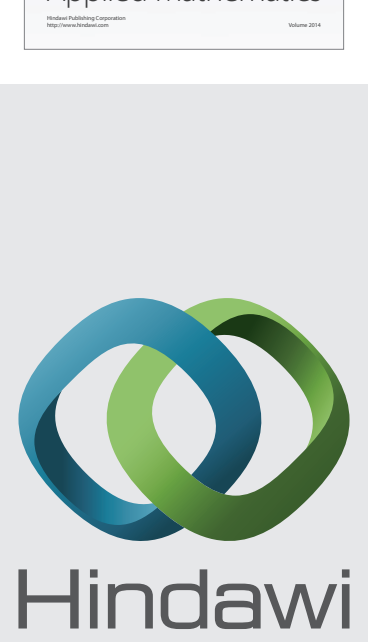

Submit your manuscripts at http://www.hindawi.com
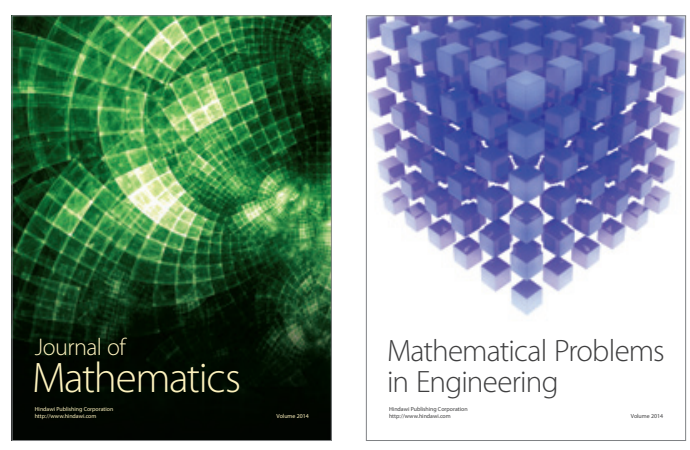

Mathematical Problems in Engineering
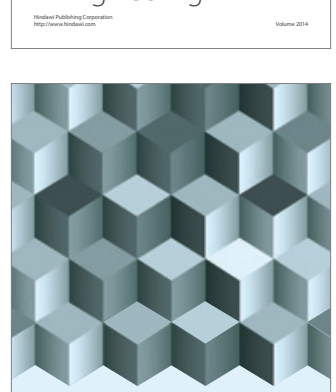

Journal of

Function Spaces
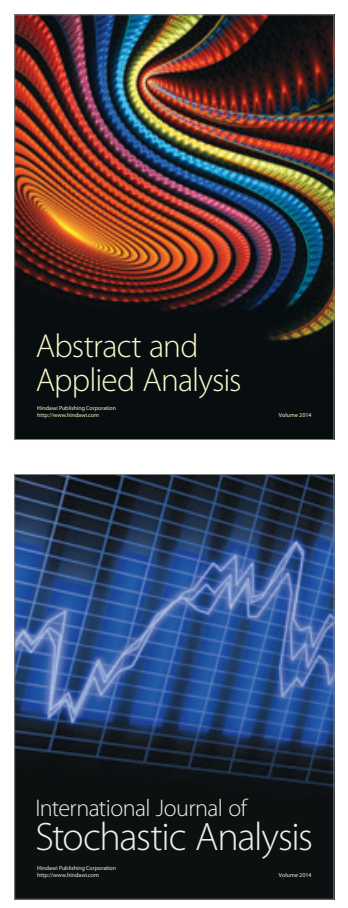

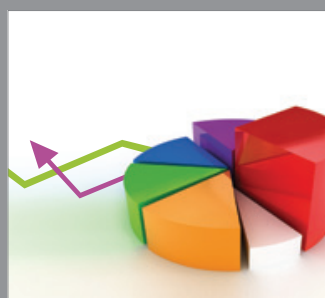

ournal of

Probability and Statistics

Promensencen
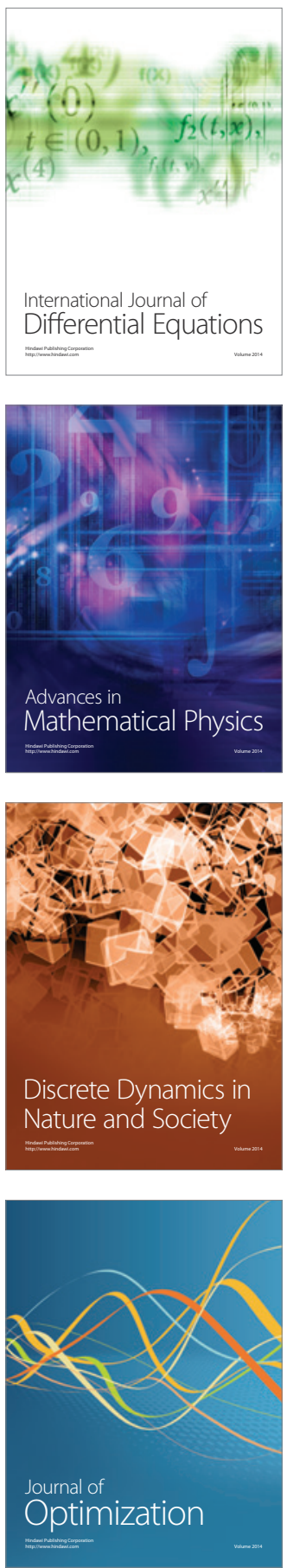